\title{
Involvement of the MAPK pathway in the pressure-induced synovial metaplasia procedure for the temporomandibular joint
}

\author{
M.J. Wu' ${ }^{1}$ H.P. Lu' ${ }^{2}$ Z.Y. Gu ${ }^{2}$ and Y.Q. Zhou ${ }^{1}$ \\ ${ }^{1}$ Department of Orthodontics, Hospital of Stomatology, \\ Zhejiang University, Hangzhou, China \\ ${ }^{2}$ School of Stomatology, Zhejiang Chinese Medical University, \\ Hangzhou, China \\ Corresponding author: Y.Q. Zhou \\ E-mail: zhouyiqunabc@126.com
}

Genet. Mol. Res. 15 (2): gmr.15027499

Received August 21, 2015

Accepted November 4, 2016

Published June 21, 2016

DOI http://dx.doi.org/10.4238/gmr.15027499

\begin{abstract}
Abnormal pressure is an important factor that contributes to bone adaptation in the temporomandibular joint (TMJ). We determined the effect of the mitogen-activated protein kinases (MAPK) pathway on the pressure-induced synovial metaplasia procedure for the TMJ, both in vitro and in vivo. Synovial fibroblasts (SFs) were exacted from rat TMJs and exposed to different hydrostatic pressures. The protein extracts were analyzed to determine the activation of ERK1/2, JNK, and p38. Surgical anterior disc displacement (ADD) was also performed on Japanese rabbits, and the proteins of TMJ were isolated to analyze pressure-induced MAPK activation after 1, 2, 4, and 8 weeks. The results showed that the activation of ERK $1 / 2$ and JNK in SFs significantly changed with increasing hydrostatic pressure, whereas p38 activation did not change. Moreover, p38 was activated in animals 1 week after surgical ADD. The levels of p38 gradually increased after 2 and 4 weeks, and then slightly decreased but remained higher than in the control 8 weeks after surgical ADD. Nevertheless,
\end{abstract}


JNK was rarely activated after the ADD treatment. Our findings suggest the involvement of MAPK activation in the pressure-induced synovial metaplasia procedure with pressure loading in TMJ.

Key words: Synovial fibroblasts; Disc displacement; Bilaminar zone; Hydrostatic pressure

\section{INTRODUCTION}

Temporomandibular joint disorder (TMD) is a common and complicated disease in oral surgery and orthodontics. Disc displacement is the main mechanism of TMD pathogenesis (Santos et al., 2013). Nevertheless, imageological evidence shows that not all patients with disc displacement exhibit obvious signs or symptoms of TMD (Kircos et al., 1987; Tallents et al., 1996; Goldstein, 1999). This phenomenon may be due to the remodeling of the temporomandibular joint (TMJ) when adapting to a new mechanical environment after disc displacement (Gu et al., 2003). Thus far, the molecular and cellular mechanisms of TMJ remodeling remain ambiguous. In our previous study, we found that the bilaminar zone (BZ) in TMJ changed from loose connective tissue to dense connective tissue, and cartilage metaplasia occurred during the pathological progress of TMD (Gu et al., 2006). The disclike adaptive alteration in the BZ may function as the disc (Gu et al., 2003). The number of chondrocytes also gradually increased, and typical cartilage formation was observed (Hall et al., 2001; Zhang et al., 2006).

Synovial fibroblasts (SFs), an important component of the BZ in TMJ, can respond to the movement of articular cartilage. As a mechanical factor, pressure shock induced by normal or abnormal movement of articular cartilage can change the arrangement of differentiation filaments and cellular function (Pedersen and Nilius, 2007; Zhang et al., 2007). During regulatory progression, most cells activate transport pathways to maintain intra-articular fluid and joint stability (Coleman et al., 1998). As such, the exact molecular events and relative pathways during TMJ remodeling after pressure loading must be investigated.

Mitogen-activated protein kinases (MAPK) exhibit a broad range of activities for controlling various cellular events. The MAPK family has three important members, namely, extracellular signal-regulated kinases (ERK)-1/2 (ERK1/2), c-Jun aminoterminal kinases (JNK), and p38 MAPK (Seger and Krebs, 1995). Studies on MAPKs in several types of cells have indicated that pathway activation occurs after loading stimulation (Hofmann et al., 2004; De Croos et al., 2006). Induction of various extracellular signal transduction pathways, which regulate morphology, proliferation, differentiation, or survival, triggers the activation of at least one MAPK (Peyssonnaux and Eychène, 2001; Volmat and Pouysségur, 2001). In the present work, we attempted to determine the effect of the MAPK pathway on the pressureinduced synovial metaplasia procedure in TMJ, both in vitro and in vivo.

\section{MATERIAL AND METHODS}

\section{Cell culture}

SFs extracted from rat TMJs were prepared according to the method described in the literature, with minor modifications (Ogura et al., 2002). Briefly, the double condyles of 
3-month-old Sprague Dawley rats ( 200 g, obtained from the Zhejiang Center of Laboratory Animals, China) were excised. The surface parts of synovial tissues were isolated from the rat TMJs. The samples were thoroughly washed with phosphate-buffered saline (PBS), minced, and cultured on a cover slip in Dulbecco's modified Eagle's medium (Gibco, Grand Island, NY, USA) supplemented with $4 \mathrm{mM}$ L-glutamine (Irvine Scientific, Santa Ana, CA, USA), antibiotics (100 U/mL penicillin, Sigma-Aldrich, St. Louis, MO; and $50 \mathrm{mg} / \mathrm{mL}$ gentamicin, Invitrogen Life Technologies), and 15\% heat-inactivated fetal calf serum (Gibco). When growth from the explants reached confluence, SFs were trypsinized and subcultured under similar conditions. We used SFs from the sixth to eighth passages for the experiments. SFs were confirmed through immunocytochemical staining to detect the marker proteins CD68 and vimentin, which are macrophage and fibroblast markers, respectively, as described in our previous study (Wu et al., 2008).

\section{Application of hydrostatic pressure}

The apparatus used in this study was designed according to the descriptions provided by Smith et al. (1996) and Yamamoto et al. (2006) with minor modifications (Smith et al., 1996; Yamamoto et al., 2006). A constant hydrostatic pressure of 30, 60, or $90 \mathrm{kPa}$ was applied for $12 \mathrm{~h}$. Cells were seeded in a tissue culture dish and placed in the same apparatus under the same conditions; these cells were used as non-pressurized controls, as reported in our previous study (Wu et al., 2008).

\section{Experimental animals}

Twenty-eight healthy adult Japanese white rabbits (from the Medical Animal Center of Zhejiang University), weighing 2-3 kg, were used in this study. The right TMJs of 20 rabbits were subjected to surgical induction of anterior disc displacement (ADD). Five of these rabbits were sacrificed at 1,2, 4, and 8 weeks after the surgery. Another five rabbits were used as shamoperation controls, and were sacrificed 4 weeks post-operation. The remaining three rabbits that had not undergone surgery were used as normal controls. All animal experiments were conducted in accordance with the guidelines of the United States National Institutes of Health with respect to the care and use of animals for experimental procedures. Animal procedures were approved by the Experimental Animal Ethics Committee of Zhejiang University.

\section{Surgical operation and rabbit ADD model}

Surgical ADD was performed on experimental and sham-operation animals according to the procedures reported in our previous study (Gu et al., 2006). The rabbits were anesthetized by an intravenous injection of amobarbital $(3 \mathrm{mg} / \mathrm{kg})$. A $3 \mathrm{~cm}$-long incision was created on the skin overlying the right zygomatic process of the squamosal bone, and the zygomaticosquamosal suture was exposed. The upper border of the anterior third of the zygomatic arch contained two small processes. A hole was drilled under the anterior process. In the inferoanterior process of the temporal root, the anterior part of the disc was vertically passed from the inferior to the superior using a needle with an elastic rubber band (TP Orthodontics, USA). The double strands of the elastic rubber band were stretched forward from $7 \mathrm{~mm}$ to 16 
$\mathrm{mm}$ and fixed in the hole using a $0.20-\mathrm{mm}$ diameter steel wire. The surgical procedure was considered successful if the elastic rubber band was taut, and there was no tissue laceration in the posterior walls of the orbit after the elastic rubber band had been fixed. The wound was closed in layers. Three animals that served as surgical controls underwent sham operations in a similar manner. The zygomatico-squamosal suture was exposed and separated, and the wound was closed in layers. Food intake was monitored every day after the surgery. Each rabbit was weighed daily during the entire experimental period.

\section{Tissue collection and sample preparation}

The rabbits were sacrificed by air embolization under general anesthesia. The right TMJs from all 25 surgical animals and bilateral TMJs from normal animals were rapidly removed. The BZ structures attached to the posterior of the disc were dissected on ice-cold glass plates. The tissues were immediately frozen on dry ice and stored at $-80^{\circ} \mathrm{C}$.

Frozen tissues were finely minced and homogenized in 10 volumes of extraction buffer $(50 \mathrm{mM}$ Tris- $\mathrm{HCl}, \mathrm{pH} 7.5,150 \mathrm{mM} \mathrm{NaCl}, 1 \mathrm{mM}$ sodium orthovanadate, $1 \mathrm{mM}$ phenylmethanesulfonyl fluoride, $1 \mathrm{mM}$ sodium fluoride, $0.5 \%$ sodium deoxycholate, $0.1 \%$ sodium dodecyl sulfate, $1.0 \%$ IGEPAL, $10 \mathrm{mg} / \mathrm{mL}$ each leupeptin, aprotinin, and pepstatin). Samples were shaken in an ice bath for $30 \mathrm{~min}$ and then centrifuged at $30,000 \mathrm{~g}$ for $20 \mathrm{~min}$. Supernatants were collected. Tissue extracts were diluted to protein concentrations of $3 \mathrm{mg} /$ $\mathrm{mL}$ (hippocampal) and $5 \mathrm{mg} / \mathrm{mL}$ (cerebral cortex) with the extraction buffer.

\section{Western blotting analysis}

The proteins extracted from different pressurized SFs and untreated controls were quantified using the modified Lowry method (Peterson, 1979). A $20-\mu \mathrm{L}$ sample containing $30 \mu \mathrm{g}$ protein was separated by sodium dodecyl sulfate polyacrylamide gel electrophoresis (SDS-PAGE), and transferred onto nitrocellulose membranes. The blots were blocked $(1 \mathrm{~h})$ with 5\% non-fat dry milk in Tris-buffered saline (TBS) at room temperature, and incubated overnight at $4{ }^{\circ} \mathrm{C}$ with antibodies against ERK1/2 (1:500), JNK (1:800), p38 (1:800) (Santa Cruz, CA, USA), phosphor-ERK, (p-ERK, 1:1000), phosphor-JNK (p-JNK, 1:1000), and phosphor-p38 (p-p38, 1:1000) (Cell Signaling Technology, Inc.) diluted with the blocking buffer. The membranes were incubated for $1 \mathrm{~h}$ with the secondary antibody (1:5000), and rinsed three times for $5 \mathrm{~min}$ in Tris-buffered saline with 0.1\% Tween-20 (TBST).

Tissue extracts prepared with equal amounts of protein were mixed 1:1 with a buffer (100 mM Tris-HCl, 4\% SDS, 20\% glycerol, 10\% 2-mercaptoethanol, $0.005 \%$ bromophenol blue, pH 6.8), and boiled for 5 min before loading on the gel with molecular weight markers. The samples were resolved on 10\% SDS-PAGE, transferred to polyvinylidene difluoride (PVDF) membranes (Millipore) by semi-dry transfer in Towbin's buffer (20\% methanol, 25 $\mathrm{mM}$ Tris, $192 \mathrm{mM}$ glycine), and blocked with 5\% skimmed milk powder in TBST. Anti-p-JNK (mouse monoclonal antibody raised against the JNK sequence containing phosphorylated Thr183 and Tyr-185, 1:300) and anti-p-p38 (mouse monoclonal antibody against p38 sequence containing phosphorylated Thr-180 and Tyr-182, 1:300). The blots were washed twice with TBST, and twice with $1 \%$ blocking solution in TBS, and then incubated with horseradish peroxidase-linked secondary antibody (goat anti-mouse; 1:2000) for $1 \mathrm{~h}$ at room temperature. 


\section{Statistical analysis}

A two-factor analysis of variance with replication was used to compare all results. Data were considered significant at $\mathrm{P}<0.05$.

\section{RESULTS}

\section{Activation of MAPK in hydrostatic pressure-treated SFs}

Western blotting analysis was performed to elucidate the activation of ERK1/2, JNK, and p38 induced by mechanical stimuli. The activation of the three proteins was related to the hydrostatic pressure, as shown in Figure 1. ERK1/2 production decreased at $30 \mathrm{kPa}$ compared with at $0 \mathrm{kPa}$ after $12 \mathrm{~h}$ of culture, peaked at $60 \mathrm{kPa}$, and then decreased at $90 \mathrm{kPa}$. JNK production increased at $30 \mathrm{kPa}$ hydrostatic pressure and then decreased at 60 and $90 \mathrm{kPa}$. Nevertheless, JNK activation was approximately constant over varying hydrostatic pressure. The production and activation of p-p38 did not change with hydrostatic pressure.
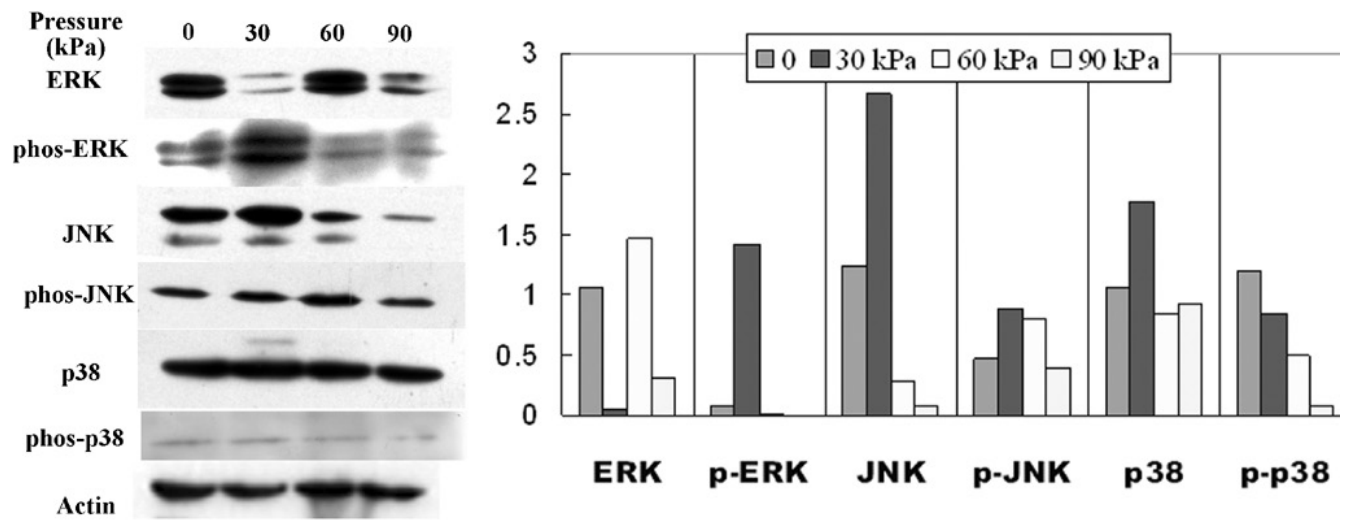

Figure 1. Western blot analysis data for ERK, JNK, and p38. ERK and p-ERK (A), JNK and p-JNK (B), p38 and phos-p38 (C), actin (D) (1, control; 2, $30 \mathrm{kPa} ; 3,60 \mathrm{kPa} ; 4,90 \mathrm{kPa})$, histogram (E) of the production levels of the three different proteins in the control, and at 30,60 , and $90 \mathrm{kPa}\left(\%\right.$ of control). ${ }^{*} \mathrm{P}<0.05,{ }^{*} * \mathrm{P}<0.01$, vs control.

\section{Effects of ADD on JNK and p38 phosphorylation in BZ}

The anti-p-JNK antibody reacted with two bands, namely, p-JNK1 at $46 \mathrm{kDa}$ and p-JNK2 at $54 \mathrm{kDa}$. Figure 2 shows that p-JNK1 was hardly detected in the BZ before or after $\mathrm{ADD}$, and p-JNK2 was only weakly detected in the early first and second week after ADD. The total expression of JNK1/2 did not change in any of the experimental groups in the BZ. A single band for phos-p38 at $38 \mathrm{kDa}$ was observed in the tissue extracts of the BZ. The production of phos-p38 did not significantly differ between the normal and sham-operation control groups, as shown in Figure 3. However, phos-p38 production was significantly enhanced compared with the normal control, and continued to increase 1 week after ADD. Moreover, phos-p38 reached its highest level at 4 weeks, and the level was approximately seven times higher than in the control. 


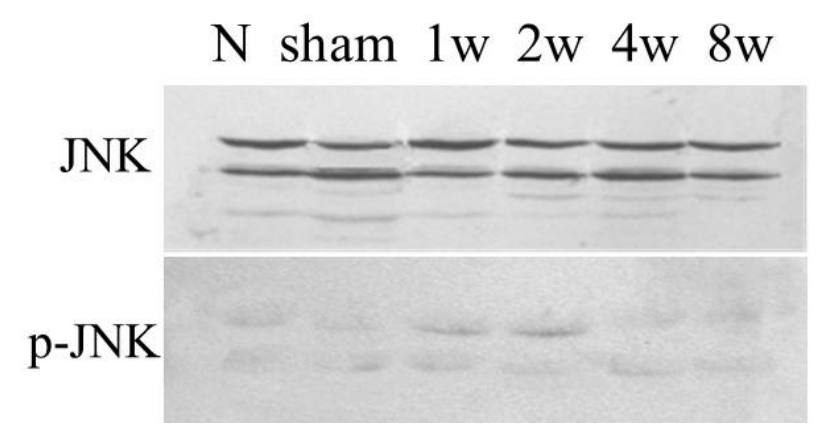

Figure 2. Activation of JNK after A disc displacement in the bilaminar zones.

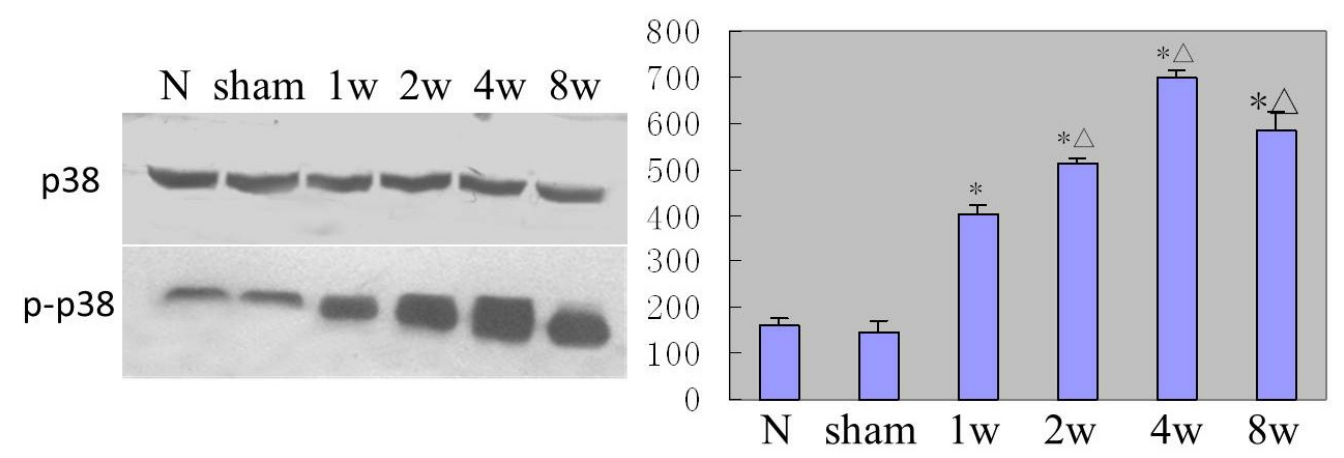

Figure 3. Activation of p38 in the bilaminar zone of the normal group (N), the sham control group (sham), and 1, 2, 4 , and 8 weeks after A disc displacement. ${ }^{*} \mathrm{P}<0.05$ vs control group; ${ }^{\wedge} \mathrm{P}<0.05$ vs the former group.

\section{DISCUSSION}

Cell adaptation is dependent on various loading conditions, such as strain magnitude, duration, frequency, type (compression, tension, or shear), and distribution. Tensile or compressive pressure is generally important for bone adaptation (Qin et al., 1996). Increasing evidence suggests that hydrostatic pressure gradients must develop within the bone to initiate bone adaptation (Duncan and Turner, 1995). The mechanical environment of SFs can be reproduced in vitro by direct compression of the articular surface of the cartilage. Pressure can modulate various interactions and destroy the cartilage and bones of patients (Voulgari et al., 2005). Many types of cell can respond to changes in mechanical pressure with specific compensatory mechanisms (Almarza and Athanasiou, 2006; Koolstra and van Eijden, 2006). Therefore, in the present study, we mimicked the dynamic environments in the TMJ in vitro by applying hydrostatic pressure. However, the microenvironment in the joint is complicated. In a real pathological condition, $\mathrm{SFs}$ of the $\mathrm{BZ}$ suffer from complex mechanical environmental conditions, including inflammation and hydrostatic pressure after ADD, which may be the reason for the inconsistent MAPK production between in vivo and in vitro assays. Based on our preliminary studies, SFs can respond to the mechanical stimuli of constant hydrostatic pressure and to the expression of osteoinductive proteins, such as TGF- $\beta$ and BMP- 2 , induced by constant hydrostatic pressure (Wu et al., 2008). 
MAPK signal transduction pathways are involved in the control of diverse cellular events, including cell proliferation, differentiation, and apoptosis (Chiri et al., 1998). ERK1/2, JNK, and p38 are activated in the rheumatoid arthritis synovium (Schett et al., 2000). ERK1/2 regulates cellular proliferation and is an essential component in the mechanotransduction process (Kapur et al., 2003). The JNK and p38 pathways are activated by inflammatory cytokines and cellular stressors, and the latter pathway plays a critical role in regulating inflammatory processes in vivo (Han et al., 1999; Adams et al., 2001). Both ERK and p38 MAPKs are activated during fibroblast contraction of stressed collagen matrices under isometric tension (Lee et al., 2000). The MAPK pathway may be a molecular mechanism for acacetin inhibition of MMPs in IL-1 $\beta$-induced FLSs (Chen et al. 2015). Based on these findings, we hypothesized that MAPK activation plays a critical role in transducing mechanical stimuli to elicit cellular responses.

Our results in vitro showed that mechanical stress induced by hydrostatic pressure involved the activation of ERK1/2, JNK, and p38 at different levels. Mechanical stimulation induced a rapid activation of ERK1/2, resulting in visible phosphorylation at $30 \mathrm{kPa}$. JNK production was modified under different hydrostatic pressures, whereas JNK activation varied little with changes in hydrostatic pressure. By contrast, changes in p38 and p-p38 were not visible. Generally, JNK and p38 are activated through distinct kinase cascades, which connect the cell surface to specific transcription factors and various regulatory proteins (Nishida and Gotoh, 1993; Seger and Krebs, 1995; Su and Karin, 1996). Thus, our findings suggest that JNK and p38 are less sensitive than ERK1/2 to changes in hydrostatic pressure.

The BZ of TMJ comprises loose connective tissues located behind the articular disc proper, which is mostly composed of collagenous and elastic fibers. The major cells in the BZ include fibroblasts, with spindly and polygonal long shuttle-shaped nuclei. The density of fibrous tissues and SFs in the superior attachment gradually increased, and the wall of blood vessels in the inferior attachment thickened, leading to a narrower cavity after ADD. The BZ was also pulled over the mandible condyle and bore abnormal loading, which converted the zone into a disc-like structure; this structure is characterized by coarse collagenous fiber bundles and scattered chondrocytes, which are surrounded by a matrix containing cartilagelike glycosaminoglycans (Mills et al., 1994; Hall et al., 2001; Gu et al., 2003; Zhang et al., 2006). Some cells in the BZ gradually transformed from SFs to chondrocyte-like cells. As in previous experiments, no significant difference were found between the sham surgery and normal control groups in the present study. ADD inflicted slight trauma to TMJ instead of opening the TMJ capsule.

Our animal experiment results showed that the activation level of p38 significantly increased with time-course changes at 1,2, and 4 weeks after ADD. Although p38 activation decreased at 8 weeks rather than at 4 weeks, the level was still significantly higher than in the control. Thus, p38 activation may be a critical regulator in transducing mechanical stimuli to elicit responses of disc-like remodeling in the BZ after ADD. The relation between p38 activation and the time-course changes after ADD may indicate that the rapid transduction to remodeling occurred soon after ADD. Moreover, the transduction to remodeling in the BZ weakened 8 weeks after ADD.

Although JNK activation is involved in mechanical stimulation and cartilage-specific gene expression in chondrocytes both ex vivo and in vitro, there is no evidence that the JNK pathway regulates chondrogenic differentiation within the embryonic mesenchyme (Oh et 
al., 2000). Nakamura et al. (1999) found that activation of the p38 pathway is important in the study of chondrogenic differentiation of ATDC5 cells induced by growth/differentiation factor (GDF)-5 (Nakamura et al., 1999). However, the JNK pathway did not participate in this process. In the present study, JNK activation was not evident in the BZ after ADD. This finding suggests that the JNK pathway plays a minor role in the process of BZ remodeling after ADD.

Mandibular condylar cartilage generally suffers pressure induced by frequent jaw movement and biting hard on food. Under physiological conditions, the activity of the TMJ is enhanced by dental occlusion. Although the pathogenesis of TMD remains unknown, local biomechanical forces exposed to articular cartilage potentially play a crucial role in the initiation of disc displacement. Intra-articular pressure varies and becomes complex when mandible movement and disc displacement occur. The BZ was evaluated to characterize remodeling in our former research. This finding implied the involvement of MAPK activation in the pressure-induced synovial metaplasia procedure with pressure loading in the TMJ. The compensatory mechanism between the appropriate pressure and the adaptive changes in TMJ can provide a biological basis for clinical therapy in patients with TMD.

\section{Conflicts of interest}

The authors declare no conflict of interest.

\section{ACKNOWLEDGMENTS}

Research supported by grants from the National Natural Science Foundation of China (grant \#81371167 and \#81200803), and the Foundation of Zhejiang Health and Family Planning Commission (\#2011KYA105). The sponsors had no role in the study design or in the collection, analysis, or interpretation of the data, the writing of the manuscript, or the decision to submit the manuscript for publication.

\section{REFERENCES}

Adams JL, Badger AM, Kumar S and Lee JC (2001). p38 MAP kinase: molecular target for the inhibition of proinflammatory cytokines. Prog. Med. Chem. 38: 1-60. http://dx.doi.org/10.1016/S0079-6468(08)70091-2

Almarza AJ and Athanasiou KA (2006). Effects of hydrostatic pressure on TMJ disc cells. Tissue Eng. 12: 1285-1294. http://dx.doi.org/10.1089/ten.2006.12.1285

Chen WP, Yang ZG, Hu PF, Bao JP, et al. (2015). Acacetin inhibits expression of matrix metalloproteinases via a MAPKdependent mechanism in fibroblast-like synoviocytes. J. Cell. Mol. Med. 19: 1910-1915. http://dx.doi.org/10.1111/ jemm. 12564

Chiri S, De Nadai C and Ciapa B (1998). Evidence for MAP kinase activation during mitotic division. J. Cell Sci. 111: 2519-2527.

Coleman PJ, Scott D, Abiona A, Ashhurst DE, et al. (1998). Effect of depletion of interstitial hyaluronan on hydraulic conductance in rabbit knee synovium. J. Physiol. 509: 695-710. http://dx.doi.org/10.1111/j.1469-7793.1998.695bm.x

De Croos JN, Dhaliwal SS, Grynpas MD, Pilliar RM, et al. (2006). Cyclic compressive mechanical stimulation induces sequential catabolic and anabolic gene changes in chondrocytes resulting in increased extracellular matrix accumulation. Matrix Biol. 25: 323-331. http://dx.doi.org/10.1016/j.matbio.2006.03.005

Duncan RL and Turner CH (1995). Mechanotransduction and the functional response of bone to mechanical strain. Calcif. Tissue Int. 57: 344-358. http://dx.doi.org/10.1007/BF00302070

Goldstein BH (1999). Temporomandibular disorders: a review of current understanding. Oral Surg. Oral Med. Oral 
Pathol. Oral Radiol. Endod. 88: 379-385. http://dx.doi.org/10.1016/S1079-2104(99)70048-X

Gu Z, Feng J, Shibata T, Hu J, et al. (2003). Type II collagen and aggrecan mRNA expression by in situ hybridization in rabbit temporomandibular joint posterior attachment following disc displacement. Arch. Oral Biol. 48: 55-62. http:// dx.doi.org/10.1016/S0003-9969(02)00158-9

Gu Z, Zhou Y, Zhang Y, Zhao S, et al. (2006). An animal model for inducing anterior disc displacement of the temporomandibular joint. J. Orofac. Pain 20: 166-173.

Hall HD, Werther JR and Gibbs SJ (2001). The mechanism of disc displacement. J. Oral Maxillofac. Surg. 59: 961-962. http://dx.doi.org/10.1053/joms.2001.26042

Han Z, Boyle DL, Aupperle KR, Bennett B, et al. (1999). Jun N-terminal kinase in rheumatoid arthritis. J. Pharmacol. Exp. Ther. 291: 124-130.

Hofmann M, Zaper J, Bernd A, Bereiter-Hahn J, et al. (2004). Mechanical pressure-induced phosphorylation of p38 mitogen-activated protein kinase in epithelial cells via Src and protein kinase C. Biochem. Biophys. Res. Commun. 316: 673-679. http://dx.doi.org/10.1016/j.bbrc.2004.02.101

Kapur S, Baylink DJ and Lau KH (2003). Fluid flow shear stress stimulates human osteoblast proliferation and differentiation through multiple interacting and competing signal transduction pathways. Bone 32: 241-251. http:// dx.doi.org/10.1016/S8756-3282(02)00979-1

Kircos LT, Ortendahl DA, Mark AS and Arakawa M (1987). Magnetic resonance imaging of the TMJ disc in asymptomatic volunteers. J. Oral Maxillofac. Surg. 45: 852-854. http://dx.doi.org/10.1016/0278-2391(87)90235-7

Koolstra JH and van Eijden TM (2006). Prediction of volumetric strain in the human temporomandibular joint cartilage during jaw movement. J. Anat. 209: 369-380. http://dx.doi.org/10.1111/j.1469-7580.2006.00612.x

Lee DJ, Rosenfeldt $\mathrm{H}$ and Grinnell $\mathrm{F}$ (2000). Activation of ERK and p38 MAP kinases in human fibroblasts during collagen matrix contraction. Exp. Cell Res. 257: 190-197. http://dx.doi.org/10.1006/excr.2000.4866

Mills DK, Daniel JC, Herzog S and Scapino RP (1994). An animal model for studying mechanisms in human temporomandibular joint disc derangement. J. Oral Maxillofac. Surg. 52: 1279-1292. http://dx.doi.org/10.1016/0278-2391(94)90051-5

Nakamura K, Shirai T, Morishita S, Uchida S, et al. (1999). p38 mitogen-activated protein kinase functionally contributes to chondrogenesis induced by growth/differentiation factor-5 in ATDC5 cells. Exp. Cell Res. 250: 351-363. http:// dx.doi.org/10.1006/excr.1999.4535

Nishida E and Gotoh Y (1993). The MAP kinase cascade is essential for diverse signal transduction pathways. Trends Biochem. Sci. 18: 128-131. http://dx.doi.org/10.1016/0968-0004(93)90019-J

Ogura N, Tobe M, Sakamaki H, Kujiraoka H, et al. (2002). Interleukin-1 beta induces interleukin-6 mRNA expression and protein production in synovial cells from human temporomandibular joint. J. Oral Pathol. Med. 31: 353-360. http:// dx.doi.org/10.1034/j.1600-0714.2002.310606.x

Oh CD, Chang SH, Yoon YM, Lee SJ, et al. (2000). Opposing role of mitogen-activated protein kinase subtypes, erk1/2 and p38, in the regulation of chondrogenesis of mesenchymes. J. Biol. Chem. 275: 5613-5619. http://dx.doi. org/10.1074/jbc.275.8.5613

Pedersen SF and Nilius B (2007). Transient receptor potential channels in mechanosensing and cell volume regulation. Methods Enzymol. 428: 183-207. http://dx.doi.org/10.1016/S0076-6879(07)28010-3

Peterson GL (1979). Review of the Folin phenol protein quantitation method of Lowry, Rosebrough, Farr and Randall. Anal. Biochem. 100: 201-220. http://dx.doi.org/10.1016/0003-2697(79)90222-7

Peyssonnaux C and Eychène A (2001). The Raf/MEK/ERK pathway: new concepts of activation. Biol. Cell 93: 53-62. http://dx.doi.org/10.1016/S0248-4900(01)01125-X

Qin YX, McLeod KJ, Guilak F, Chiang FP, et al. (1996). Correlation of bony ingrowth to the distribution of stress and strain parameters surrounding a porous-coated implant. J. Orthop. Res. 14: 862-870. http://dx.doi.org/10.1002/ jor. 1100140604

Santos KC, Dutra ME, Warmling LV and Oliveira JX (2013). Correlation among the changes observed in temporomandibular joint internal derangements assessed by magnetic resonance in symptomatic patients. J. Oral Maxillofac. Surg. 71: 1504-1512. http://dx.doi.org/10.1016/j.joms.2013.04.033

Schett G, Tohidast-Akrad M, Smolen JS, Schmid BJ, et al. (2000). Activation, differential localization, and regulation of the stress-activated protein kinases, extracellular signal-regulated kinase, c-JUN N-terminal kinase, and p38 mitogenactivated protein kinase, in synovial tissue and cells in rheumatoid arthritis. Arthritis Rheum. 43: 2501-2512. http:// dx.doi.org/10.1002/1529-0131(200011)43:11<2501::AID-ANR18>3.0.CO;2-K

Seger R and Krebs EG (1995). The MAPK signaling cascade. FASEB J. 9: 726-735.

Smith RL, Rusk SF, Ellison BE, Wessells P, et al. (1996). In vitro stimulation of articular chondrocyte mRNA and extracellular matrix synthesis by hydrostatic pressure. J. Orthop. Res. 14: 53-60. http://dx.doi.org/10.1002/ jor. 1100140110 
Su B and Karin M (1996). Mitogen-activated protein kinase cascades and regulation of gene expression. Curr. Opin. Immunol. 8: 402-411. http://dx.doi.org/10.1016/S0952-7915(96)80131-2

Tallents RH, Katzberg RW, Murphy W and Proskin H (1996). Magnetic resonance imaging findings in asymptomatic volunteers and symptomatic patients with temporomandibular disorders. J. Prosthet. Dent. 75: 529-533. http:// dx.doi.org/10.1016/S0022-3913(96)90458-8

Volmat V and Pouysségur J (2001). Spatiotemporal regulation of the p42/p44 MAPK pathway. Biol. Cell 93: 71-79. http:// dx.doi.org/10.1016/S0248-4900(01)01129-7

Voulgari PV, Papazisi D, Bai M, Zagorianakou P, et al. (2005). Laryngeal involvement in rheumatoid arthritis. Rheumatol. Int. 25: 321-325. http://dx.doi.org/10.1007/s00296-005-0594-x

Wu MJ, Gu ZY and Sun W (2008). Effects of hydrostatic pressure on cytoskeleton and BMP-2, TGF-beta, SOX9 production in rat temporomandibular synovial fibroblasts. Osteoarthritis Cartilage 16: 41-47. http://dx.doi. org/10.1016/j.joca.2007.05.024

Yamamoto T, Kita M, Kimura I, Oseko F, et al. (2006). Mechanical stress induces expression of cytokines in human periodontal ligament cells. Oral Dis. 12: 171-175. http://dx.doi.org/10.1111/j.1601-0825.2005.01179.x

Zhang YK, Gu ZY and Hu QG (2006). Experimental study of the mechanism of chondroid metaplasia of the bilaminar zone following anterior disc displacement of temporomandibular joint. Shanghai Kou Qiang Yi Xue 15: 48-51.

Zhang Z, Kindrat AN, Sharif-Naeini R and Bourque CW (2007). Actin filaments mediate mechanical gating during osmosensory transduction in rat supraoptic nucleus neurons. J. Neurosci. 27: 4008-4013. http://dx.doi.org/10.1523/ JNEUROSCI.3278-06.2007 